\title{
1 The Heliospheric Current Sheet and Plasma Sheet 2 during Parker Solar Probe's First Orbit
}

3

\begin{abstract}
B. Lavraud ${ }^{1}$, N. Fargette ${ }^{1}$, V. Réville ${ }^{1}$, A. Szabo ${ }^{2}$, J. Huang ${ }^{3}$, A. P. Rouillard ${ }^{1}$, N. Viall ${ }^{2}$, T. D. Phan ${ }^{4}$, J. C. Kasper $^{3}$, S. D. Bale ${ }^{4,5}$, M. Berthomier ${ }^{6}$, J. W. Bonnell ${ }^{4}$, A. W. Case ${ }^{7}$, T. Dudok de Wit ${ }^{8}$, J. P. Eastwood ${ }^{9}$, V. Génot ${ }^{1}$, K. Goetz ${ }^{10}$, L. S. Griton ${ }^{1}$, J. S Halekas ${ }^{11}$, P. Harvey ${ }^{4}$, R. Kieokaew ${ }^{1}$, K. G. Klein ${ }^{12}$, K. E. Korreck ${ }^{6}$, A. Kouloumvakos ${ }^{1}$, D. E. Larson ${ }^{4}$, M. Lavarra ${ }^{1}$, R. Livi ${ }^{4}$, P. Louarn' ${ }^{1}$, R. J. MacDowall ${ }^{2}$, M. Maksimovic ${ }^{13}$, D. Malaspina ${ }^{14}$, T. Nieves-Chinchilla ${ }^{2}$, R. F. Pinto ${ }^{1}$, N. Poirier ${ }^{1}$, M. Pulupa ${ }^{4}$, N. E. Raouafi ${ }^{15}$, M. L. Stevens ${ }^{7}$, S. Toledo-Redondo ${ }^{1,16}$, and P. L. Whittlesey ${ }^{4}$
\end{abstract}

(1) IRAP, CNRS, UPS, CNES, Université de Toulouse, Toulouse, France,

(2) NASA Goddard Space Flight Center, Greenbelt, United States,

(3) University of Michigan, Ann Arbor, United States

(4) Space Sciences Laboratory, University of California, Berkeley, United States

(5) Physics Department, University of California, Berkeley, United States

(6) Laboratoire de Physique des Plasmas, Ecole Polytechnique, France

(7) Smithsonian Astrophysical Observatory, Cambridge, United States

(8) LPC2E, CNRS and University of Orléans, Orléans, France

(9) Imperial College London, Physics, London, United Kingdom

(10) University of Minnesota, Minneapolis, United States

(11) Department of Physics and Astronomy, University of lowa, lowa City, United States

(12) Lunar and Planetary Laboratory, University of Arizona, Tucson, United States

(13) LESIA, Observatoire de Paris, Meudon, France

(14) Laboratory for Atmospheric and Space Physics, University of Colorado, Boulder, United States

(15) Johns Hopkins University, Baltimore, United States

(16) Department of Electromagnetism and Electronics, University of Murcia, Murcia, Spain

\section{Abstract}

We present Heliospheric Current Sheet (HCS) and Plasma Sheet (HPS) observations during Parker Solar Probe's (PSP) first orbit around the Sun. We focus on the eight intervals that display a true sector boundary (TSB; based on suprathermal electron pitch angle distributions) with one or several associated current sheets. The analysis shows that (1) the main density enhancements in the vicinity of the TSB and HCS are typically associated with electron strahl dropouts, implying magnetic disconnection from the Sun, (2) the density enhancements are just about twice that in the surrounding regions, suggesting mixing of plasmas from each side of the HCS, (3) the velocity changes at the main boundaries are either correlated or anticorrelated with magnetic field changes, consistent with magnetic reconnection, (4) there often exists a layer of disconnected magnetic field just outside the high-density regions, in agreement with a reconnected topology, (5) while a few cases consist of short-lived density and velocity changes, compatible with short-duration reconnection exhausts, most events are much longer and show the presence of flux ropes interleaved with higher- $\beta$ regions. These findings are 
consistent with the transient release of density blobs and flux ropes through sequential magnetic reconnection at the tip of the helmet streamer. The data also demonstrate that, at least during PSP's first orbit, the only structure that may be defined as the HPS is the density structure that results from magnetic reconnection, and its by-products, likely released near the tip of the helmet streamer.

\section{Introduction}

The fast solar wind is known to come from open solar magnetic field regions in coronal holes (e.g., Cranmer 2009), while the source of the slow solar wind is less clear (e.g., Fisk et al. 1999, Tu et al., 2005, Kasper et al. 2007, 2012, Suess et al. 2009, Higginson et al. 2017). The heliospheric current sheet (HCS) is embedded in the slow solar wind. It is defined as the current sheet that extends into the heliosphere from the tip of the closed coronal magnetic field of the helmet streamer, and separates regions that connect magnetically to the two hemispheres of the Sun (e.g. Gosling et al. 1981). The location where the strahl, a suprathermal electron population permanently emitted outward from the Sun, switches from propagating parallel to anti-parallel (or vice-versa) along magnetic field lines in each hemisphere is called the true sector boundary (TSB, e.g., Kahler \& Lin, 1994, 1995, Szabo et al., 1999). Whilst in principle the HCS and TSB should be collocated, often they are not, likely as the result of interchange reconnection between open and closed field lines near the helmet streamer (e.g., Crooker et al., 2004, Huang et al., 2016). Sometimes the term HCS is used in a broader sense and embeds both the TSB and the one, or several, current sheets sustaining the radial magnetic field change near the TSB. Finally, the heliospheric plasma sheet (HPS) is a high density and high- $\beta$ region that typically surrounds the HCS, but its origin and properties are still debated (e.g., Burlaga et al. 1990, Crooker et al., 1993, 1996, 2004, Bavassano et al., 1993, Winterhalter et al. 1994, Wang et al., 1998, 2000, Liu et al. 2014) ( $\beta$ is the ratio of thermal to magnetic pressures).

Early remote-sensing observations revealed the transient release of density blobs from the tip of the helmet streamer (Sheeley et al. 1997). Since then, remote sensing and in situ observations have aimed to characterize the density enhancements, and their substructure, including their possible relation to magnetic flux ropes and solar wind type (Kasper et al. 2007, 2012, Rouillard et al., 2010a,b, Viall et al., 2010, Viall \& Vourlidas, 2015, Kepko et al. 2016, Huang et al., 2017, Sanchez-Diaz et al. 2017a,b, Di Mattteo et al., 2019). The relation between blobs and flux ropes was in particular supported by the inward plasma motions observed in association with the outward release of large blobs in remote-sensing observations (Sanchez-Diaz et al. 2017a,b). It is on this basis, and by comparing in situ data at $1 \mathrm{AU}$ and $0.35 \mathrm{AU}$, that Sanchez-Diaz et al. (2019) proposed a model for the sequential release of flux ropes by magnetic reconnection at the tip of the helmet streamer (cf. section 4 for more details). The release of flux ropes near the tip of the helmet streamer is also supported by global modeling (Higginson \& Lynch 2018), as well as laboratory experiments (Peterson et al. 2019). 
The purpose of the present paper is to refine the model of Sanchez-Diaz et al. (2019), including the description and nature of the HPS, on the basis of the new Parker Solar Probe (PSP; Fox et al. (2016)) data acquired during its first orbit around the Sun. This study is also a follow-on to that by Szabo et al. (2020), who recently investigated the same set of HCS crossings by PSP, but who mainly focused on the differences between PSP measurements in the inner heliosphere and Wind at 1 AU. They concluded in particular that the small structures (blobs and flux ropes) observed near the HCS evolve significantly as they travel from the corona to $1 \mathrm{AU}$, changing both in size and plasma properties.

\section{PSP instruments and first orbit modelling}

PSP was launched in August 2018 into a highly elliptical orbit around the Sun. The first PSP orbit already came closer to the Sun than any past mission, down to 0.165 AU. PSP comprises a set of in situ instruments that are used for the present study. We use particle data from the SWEAP (Solar Wind Electrons Alphas and Protons; Kasper et al. (2016)) instrument suite and magnetic field data from the FIELDS instrument suite (Bale et al. 2016). For SWEAP we show ion data (moments) from the Solar Probe Cup (SPC) (Case et al. 2020) and electron pitch angle distributions from the Solar Probe ANalyzers (SPANs) (Whittlesey et al. 2020). Electron pitch angles are calculated in the plasma frame using SPC velocity. We use RTN coordinates throughout the paper.

Figure 1a shows PSP orbit mapping to a distance of 5 solar radii (Rs) during its first orbit. The background color map shows the radial magnetic field (in Gauss) at 5 Rs, highlighting the expected location of the HCS (thick black line). The thin black line presents the PSP orbit mapping using a simple Parker spiral geometry while the colored points show that based on magnetic connection within the global coronal and heliospheric magnetohydrodynamics simulation of Réville et al. (2020) (note that PSP orbit goes from right to left). Figure $1 \mathrm{~b}$ displays the magnetic footpoints of PSP on the photosphere in the global simulation, with the background color map corresponding to synthetic coronal UV emissions at $193 \AA$. The modeling uses as inner boundary conditions an ADAPT map derived from GONG magnetic field at the photosphere on 6 November 2018 at 12:00 UT. As already shown in Réville et al. (2020), and confirmed with other models (Bale et al. 2019, Réville et al. 2020, Szabo et al. 2020, Badman et al. 2020), global modelling for this period overall performs well, albeit sometimes with significant errors in the timing of HCS crossings.

\section{PSP observations}

\subsection{Overview of orbit 1}

Table 1 provides the list of all eight true sector boundaries (TSB), defined as the main periods when the directionality of $315 \mathrm{eV}$ strahl electron pitch angle (PA) distribution 
switches direction (from field-aligned at $0^{\circ} \mathrm{PA}$ to anti-field-aligned at $180^{\circ}$, or vice-versa). We specifically searched for HCS and HPS signatures in the vicinity of the TSB because we want to make sure that the density signatures observed are not related to other structures (CME, stream interaction regions, etc.). By definition, the HCS and HPS are expected to exist near the TSB (e.g., Winterhalter et al. 1994; Crooker et al. 1996, 2004).

Figure 2 presents PSP in situ observations around its first perihelion between 27 October 2018 and 18 November 2018. Panels (a) and (b) show overall increases in magnetic field magnitude and density centered as expected around perihelion on 6 November 2018. The radial velocity component in panel (c) shows that PSP was primarily in the slow solar wind during the orbit, except after 15 November 2018 when significantly faster solar wind was measured. Of interest during this interval are the three TSBs crossings marked with vertical dashed lines. They are seen in panel (d) as a switch in the directionality of the $315 \mathrm{eV}$ strahl electrons from field-aligned to anti-field-aligned or vice-versa. Figure 2 is zoomed in near perihelion for clarity, but a few other TSBs sampled during the first orbit are listed in Table 1. The three TSBs marked in Figure 2 are associated with both an HCS, which consists of a clear switch in the radial magnetic field component (black line in panel (a)) from sunward to anti-sunward or vice-versa, and an HPS, observed as significant density enhancements (panel (b)) in the vicinity of the TSB and HCS.

We also note a few probable partial crossings of the HCS and HPS during the first PSP orbit. A significant one, for example, is on 30 October 2018 as marked with a red arrow in Figure $2 \mathrm{~b}$. They are not listed in Table 1 because they are not associated with TSBs. Also, the density enhancements marked with black arrows on 31 October 2018 and 12 November 2018 correspond to the two main CMEs observed during the first orbit (Korreck et al. 2020, Nieves-Chinchilla et al. 2020, Zhao et al. 2020, Giacalone et al. 2020, Mitchell et al. 2020). These density structures are thus not considered here.

\subsection{TSB, HCS and HPS during PSP first orbit}

Figure 3 shows PSP observations for interval \#4 in Table 1, on 28 October 2018, corresponding to the first vertical dashed line in Figure 2. The beginning of the interval corresponds to a "toward" sector with negative radial magnetic field (panel (d)) and antiparallel strahl electrons (panel (g)), while the last part of the interval corresponds to an "away" sector with opposite radial magnetic field and strahl directionality. Rather than being characterized by a sharp transition (e.g., case \#2 in Table 1), the change from one sector to the other is interspersed with regions of large density (panel (b)) and low magnetic field (panel (d)), and thus large $\beta$ values (panel (a)).

The key observation in Figure 3 is that the intervals of density and $\beta$ enhancement correspond systematically to magnetic field decreases and strahl dropouts. The strahl dropouts demonstrate that these regions are disconnected from the Sun (e.g. Gosling et al., 2005a), and therefore that they were produced by magnetic reconnection, likely at the tip of the helmet streamer. Additional and consistent observations are as follows: (1) the density is typically increased by nearly a factor of 2 within these regions, as compared to 
the surroundings, consistent with these intervals being reconnection exhausts created from the mixing of the plasmas from both sides of the HCS, as first found in the solar wind by Gosling et al. (2005b); (2) the magnetic field decreases are consistent with conversion of magnetic energy by magnetic reconnection; (3) the velocity and magnetic field components are typically correlated upon entrance from the toward sector into the high density region (i.e., compare for instance $V_{R}$ in panel (c) with $B_{R}$ in panel (d) across the first dashed line at 03:00 UT on 28 October 2018), while they are anti-correlated upon exit into the away sector (as is the case for all partial exits at the other dashed lines, which correspond to back and forth motions across the boundary on the same side of the HPS), as expected also for a reconnection exhaust (e.g., Gosling et al. 2005b, Phan et al. 2020); (4) there is evidence for flux rope structures, in particular between about 04:00 and 06:00 UT on 28 October 2018, and around 10 UT as well (both marked in Figure 3, and highlighted with orange shading); and (5) when the density increases the radial velocity component generally enhances compared to the adjacent solar wind, although it is not always the case in particular in the vicinity of the flux-rope structures (as expected if formed between multiple X lines, as discussed in section 4).

The two flux rope-type structures have larger magnetic fields with significant rotations, slightly lower $\beta$, and show suprathermal electron properties that are different from the surrounding. Suprathermal electrons in the flux ropes have larger fluxes and more bidirectional character than the highest $-\beta$ intervals, which always show strong strahl dropouts. Electrons PA in the flux ropes also contrast with the stronger and unidirectional fluxes of the adjacent open magnetic fields in either the away or toward sectors. It should be noted that the interval in between the two flux ropes marked in Figure 3 has a smooth magnetic field and may also be associated to the crossing of a flux rope at some distance from its main axis (given the lack of rotation and total magnetic field enhancement). However, such a possibility is not the scope of the present paper. Finally, between 15:00 UT and 22:00 UT on 28 October 2018 there are several small intervals with strahl dropouts (disappearance of the strahl at $0^{\circ}$ ) but no significant density increases. This period is shaded green in Figure 3 and discussed in section 4 .

We surveyed all TSB crossings during PSP's first orbit and analyzed their main characteristics in a similar way to the above case study. All events listed in Table 1 exhibit the following properties: the density and $\beta$ enhancements observed near the TSB, and that would be traditionally defined as the HPS, have properties consistent with that of a reconnection exhaust (and its by-products) and are mostly disconnected from the Sun through magnetic reconnection ("mostly" here relates to the fact that the flux ropes within the HPS can have different topologies, as discussed later).

\section{Discussion}

We identified HCS and HPS signatures in the vicinity of the TSB during PSP's first orbit. 
structures (CME, stream interaction regions, etc.) but are really associated with the TSB where HCS and HPS are expected, by definition (e.g., Winterhalter et al. 1994, Crooker et al. 1993, 2004).

Apart from event \#8 (for which the data is rather complex), all intervals show (1) density enhancements, generally just about twice that measured in the adjacent regions, (2) correlation and anti-correlation between velocity and magnetic field at the boundaries with the toward and away sectors, respectively (cf. previous section for details), and (3) electron strahl dropouts (but see discussion on flux ropes). These facts demonstrate that these high-density regions are magnetic reconnection exhausts mostly disconnected from the Sun. During most intervals the HCS is not a simple current sheet (cf. Table 1). It rather consists of a complex exhaust embedding a succession of high- $\beta$ regions and somewhat lower- $\beta$ flux ropes that are proposed to be the by-products of sequential magnetic reconnection at the tip of the helmet streamer, similar to the model Sanchez-Diaz et al. (2019). Szabo et al. (2020) also analyzed all TSBs and HCSs during PSP's first orbit. Although they mostly focused on the comparison with Wind data at $1 \mathrm{AU}$, suggesting that small solar wind structures suffer from significant evolution during their propagation to $1 \mathrm{AU}$, their interpretation is also that the HCS is more complex than a single and sharp current sheet.

Such observations were permitted thanks to PSP's approach close to the Sun, which allowed the observation of a mostly uncompressed and radial magnetic field topology. Although it is also a function of the HCS tilt angle, in general this contrasts with the more compressed Parker spiral at 1 AU that only allows spacecraft trajectory to cross the HCS plane at large angles, and which generally precludes the spacecraft from residing for a substantial amount of time in the vicinity of the HCS (cf. also Helios observations in Sanchez-Diaz et al. (2019)).

We noted several intervals between 15:00 and 22:00 UT on 28 October 2018 (greenshaded area in Figure 3) with strahl dropouts unrelated to density increases or magnetic field decreases. Similar intervals are seen in other events as well. They are consistent with the magnetic reconnection scenario. Indeed, if the high-density regions are the exhausts from reconnection at the tip of the helmet streamer, one expects the exhaust magnetic field to thread through its boundaries, thus forming a thin layer just outside the exhaust that would show a disconnected topology as well. This is akin to the magnetosheath boundary layer formed by reconnection just outside the Earth's magnetopause (e.g., Fuselier et al., 1995, Lavraud et al., 2006), and was previously observed in the solar wind as well (Lavraud et al. 2009).

Figure 4, adapted from Sanchez-Diaz et al. (2019), summarizes some implications of the present study. Sanchez-Diaz et al. (2017ab, 2019) proposed that density blobs and flux ropes are released periodically from the tip of helmet streamers with a periodicity of 10-20 hours, in agreement with white-light observations of density blobs (e.g. Sheeley et al. 2009, Rouillard et al. 2010a). They also showed the frequent observation of smaller density and magnetic structures. Such smaller-scale structures are more compatible with 
a periodicity on the order of 1-3 hours as found in the analysis of solar wind density fluctuations (e.g., Viall et al., 2010, Viall \& Vourlidas, 2015, Kepko et al. 2016). In the present PSP observations, the duration of the flux ropes (from all events) ranges grossly from 1 to $4 \mathrm{~h}$ (a $2 \mathrm{~h}$ long structure at $300 \mathrm{~km} / \mathrm{s}$ corresponds to a size of $\sim 3 \mathrm{Rs}$ ). Such scale sizes are more comparable with the latter quasi-periodic structures (e.g., Kepko et al. 2016).

The present analysis suggests that the HPS is a high-density region whose nature is essentially a large reconnection exhaust mostly disconnected from the Sun (blue field lines in Figure 4). Together with past works, it also suggests that it is composed of a succession of high- $\beta$ blobs (dark grey regions) and flux ropes, and that there exists a large spectrum in the size of the flux ropes that may be released through sequential magnetic reconnection above the helmet streamers (from tens of minutes to few tens of hours). Future statistical works with in situ observations, combined with modeling, are needed to determine what drives these different scales and periodicities, and their association to magnetic reconnection near the tip of the helmet streamer.

The flux ropes identified in all events show variable electron strahl properties. They 253 often show the presence of residual fluxes which mark a different connectivity to the Sun. While the two flux ropes in Figure 3 show bidirectional strahl electrons, albeit with rather low fluxes, it is known from 1 AU observations that flux ropes near the HCS can show various connectivities to the Sun (e.g., Kilpua et al. 2009, Rouillard et al. 2011, SanchezDiaz et al. 2019). In Figure 4, the black field lines threading through the flux ropes are anchored on the Sun at only one end. This Figure was adapted from Sanchez-Diaz et al. (2019) and reflects the fact that flux ropes near the HCS at 1 AU often show unidirectional strahl electrons. Yet, as found in past studies at 1 AU (e.g., Sanchez-Diaz et al. 2019) and confirmed here by the rather bidirectional strahl electrons in the flux ropes of Figure 3, the magnetic field within the flux ropes may also be anchored on the Sun at both ends. This possibility is depicted with a red dashed line in Figure 4 . There might be in principle four different topologies possible within the flux ropes as a function of the length and properties of the X lines formed: fully disconnected (strahl dropout), anchored at the Sun at both ends (bidirectional strahl), anchored at the Sun at only one end in either hemisphere (either parallel or anti-parallel strahl). An analogy is here drawn with studies of flux ropes at the Earth's magnetopause (Pu et al. 2013), but further studies remain to be performed to determine whether this analogy is plausible at the Sun. We note that recent global simulations by Higginson \& Lynch (2018) have been able to reproduce complex topologies for flux ropes created at the tip of the helmet streamer.

Szabo et al. (2020) recently noted that high density regions are less prominent and frequent in PSP observations compared to $1 \mathrm{AU}$ (using Wind data for the same period). On the other hand, Crooker et al. (2004) found that the high- $\beta$ plasma sheet is shorter than the high-density plasma sheet at $1 \mathrm{AU}$. We propose that the high- $\beta$ plasma sheet is the main plasma sheet, as observed here, and resulting from magnetic reconnection at the tip of the helmet streamer. As plasma evolves during propagation in a Parker Spiral configuration additional compression near the HCS will lead to a broader high-density 
region around the original HPS. Magnetic reconnection produces a high $\beta$ exhaust because it increases density and temperature but decreases magnetic field at the same time. By contrast, adiabatic compression, which may occur around the HPS during propagation, increases both thermal plasma and magnetic pressures. In the end, this process may produce a broad high-density region around the thinner high- $\beta$ HPS initially created by magnetic reconnection at the tip of the helmet streamer.

Our scenario has similarities with that described by Wang et al. $(1998,2000)$ (cf. also Crooker et al. 1993, 1996), who proposed that the entire HPS consists of discontinuous plasma parcels. Yet, the present observations suggest the full disconnection of most of the HPS, through magnetic reconnection at the tip of the helmet streamer, rather than the transient release of material from closed magnetic loops through interchange reconnection with the adjacent open magnetic fields (Wang et al. 1998, 2000, Crooker et al. 2004). The reason is that interchange reconnection implies the magnetic field lines remain attached to the Sun at one end, and thus contain an electron strahl population (rather than a dropout). While this is not what the present observations suggest, it does not preclude the occurrence of interchange reconnection in other contexts or at other times. It also does not preclude interchange reconnection to form structures in the nearby slow solar wind, such as the famous jets and switchbacks reported with PSP (Kasper et al. 2019, Bale et al. 2019), or even most of the background slow solar wind (e.g., Fisk et al. 1999, Fisk \& Schwadron 2001). It also does not preclude interchange reconnection to be related to the observation of strahl electrons in the flux ropes, as suggested by the flux rope topology in the simulations by Higginson \& Lynch (2018).

Finally, we wish to note that the notion of plasma sheet for the heliosphere and Earth's magnetosphere display both similarities and differences. The main analogy is that both plasma sheets are in gross equilibrium at large scales, with a higher- $\beta$ plasma sheet surrounded by stronger magnetic fields in the lobes/open field regions. In both cases magnetic reconnection may occur in a transient fashion at the tip of the closed field region, leading to the sequential release of flux ropes. The main difference between the two plasma sheets, on the other hand, is that the plasma sheet at Earth is primarily formed by the loading of plasma from the solar wind either from the flanks or through the lobes via the Dungey cycle (Dungey, 1961). By contrast, there is no such filling mechanism in the case of the Sun and thus there is no pre-existing plasma sheet. The way to form a plasma sheet in this context is through the mechanism depicted here.

\section{Conclusions}

Based on a survey of eight TSB intervals during PSP's first orbit, we found that the HCS was typically not a single and sharp current sheet but was instead a broad region composed of a complex succession of high- $\beta$ blobs and flux ropes, consistent with the periodic release of flux ropes through sequential magnetic reconnection at the tip of the 
helmet streamer. Based on this and past works, this process is likely capable of producing a large spectrum of flux ropes sizes, from tens of minutes to few tens of hours.

The present observations also demonstrate that during PSP's first orbit the HPS, identified as a high density and high- $\beta$ region in the vicinity of the TSB and HCS, is solely defined as the exhaust region produced by magnetic reconnection, likely at the tip of the helmet streamer. There is simply no other structure during this orbit that may be defined as the HPS.

There is no doubt that the next PSP orbits, combined with Solar Orbiter observations and modeling, will help determine whether these findings are always valid, and whether there exist other types of HCS structures or other origins to the HPS.

\section{Acknowledgements}

Work at IRAP was performed with the support of CNRS and CNES. We visualize data using the CL software available at http://clweb.irap.omp.eu/, developed by E. Penou. Parker Solar Probe was designed, built, and is now operated by the Johns Hopkins Applied Physics Laboratory as part of NASA's Living with a Star (LWS) program (contract NNN06AA01C). Support from the LWS management and technical team has played a critical role in the success of the Parker Solar Probe mission. All the data used in this work are available on the SWEAP (http://sweap.cfa.harvard.edu/data/) and FIELDS (http://fields.ssl.berkeley.edu/data/) data archives. JPE acknowledges support from UK grant UKRI/STFC ST/N000692/1.

\section{References}

Badman, S. T., Bale, S. D., Martínez Oliveros, J. C., et al 2020, ApJS, 246, 23

Bale, S. D., Goetz, K., Harvey, P. R., et al. 2016, SSRv, 204, 49

Bale, S. D., Badman, S. T., Bonnell, J. W., et al. 2019, Nature, 576237

Bavassano, B., Woo, R., \& Bruno, R. 1997, GRL, 24, 1655-1658.

Boerner, P., et al. 2012, SoPh, 275, 41-66

Burlaga, L. F., Scudder, J. D., Klein, L. W., \& P. A. Isenberg 1990, JGR, 95, 2229-2239.

Case, A. W., Kasper, J. C., Stevens, M. L., et al. 2020, ApJS, 246, 43

Cranmer, S. R. 2009, Living Reviews in Solar Physics, 6, 1

Crooker, N. U., Siscoe, G. L., Shodhan, S. et al. 1993, JGR, 98, 9371- 9381.

Crooker, N. U., Burton, M. E., Siscoe, G. L., et al. 1996, JGR, 101, A11, 24331

Crooker, N. U., Huang, C. L., Lamassa, S. M., et al. 2004, JGR, 109, A03107

Di Matteo, S, Viall, N. M., Kepko, L., et al. 2019, JGR, 124, 2, 837

Dungey, J. W. 1961, PRL, 6, 47

Fisk, L. A., Zurbuchen, T. H., \& Schwadron, N. A. 1999, ApJ, 521, 868 
351

352

353

354

355

356

357

358

359

360

361

362

363

364

365

366

367

368

369

370

371

372

373

374

375

376

377

378

379

380

381

382

383

384

385

Fisk, L., \& Schwadron, N. 2001, ApJ, 560, 425

Fox, N. J. et al. 2016, SSR, 204, 7-48

Fuselier, S. A., Anderson, B. J., Onsager, T. G. 1995, JGR, 100, 11,805.

Giacalone, J., et al 2020, ApJS, 246; 29

Gosling, J. T., Borrini, G., Asbridge, J. R., et al., 1981, JGR, 86, 5438- 5448.

Gosling, J. T., Skoug, R. M., McComas, D. J. \& Smith, C. W. 2005a, GRL, 32, 05105

Gosling, J. T., Skoug, R. M., McComas, D. J. \& Smith, C. W. 2005b, JGR, 110, A01107

Higginson, A., Antiochos, S., DeVore, C., Wyper, P., \& Zurbuchen, T. 2017, ApJL, 840, L10

Higginson, A. K., \& Lynch, B. J. 2018, ApJ, 859, 6

Huang, J., Liu, Y. C.-M., Qi, Z., et al. 2016, 121, 10,768-10,782

Huang, J., Liu, Y. C.-M., Peng, J. et al. 2017, JGR ,122, 6927-6939

Kahler, S., \& Lin, R. P. 1994, GRL, 21, 1575- 1578

Kahler, S., \& Lin, R. P. 1995, SoPh, 161, 183- 195.

Kasper, J. C., Stevens, M. L., Lazarus, et al. 2007, ApJ, 660, 901

Kasper J. C., Stevens, M. L., Korreck, K. E. et al. 2012, ApJ, 745,162

Kasper, J. C., Abiad, R., Austin, G., et al. 2016, SSRv, 204, 131

Kasper, J. C., Bale, S. D., Belcher, J. W., et al. 2019, Nature, 576, 228

Kepko, L., Viall, N. M., Antiochos, S. K., et al. 2016, GeoRL, 43, 4089

Kilpua, E. J. K., Luhmann, J. G., \& Gosling, J. 2009, SoPh, 256, 327

Lavraud, B., Thomsen, M. F., Lefebvre, B. et al. 2006, JGR, 111, A05211

Lavraud, B., Gosling, J. T. Rouillard, A. P. et al. 2009, SoPh, 256, 1-2, 379-392

Liu, Y. C. -M., Huang, J., Wang, C. et al., 2014, JGR, 119, 8721

Mitchell, D. G., Giacalone, J., Allen, R. C., et al. 2020, ApJS, 246, 59

Peterson, E. E., et al. 2019, Nature Phys., 15, 1095

Phan, T.-D., et al. 2020, ApJS, 246, 34

Pu, Z. Y., Raeder J., Zhong, J. et al. 2013, GRL, 40, 3502-3506

Rouillard, A. P., Davies, J. A., Lavraud, B. et al. 2010a, JGR, 115, A04103

Rouillard, A. P., Davies, J. A., Lavraud, B., et al. 2010b, JGR, 115, A04104

Sanchez-Diaz, E., Rouillard, A. P., Davies, J. A. et al., 2017a, ApJ, 851, 32

Sanchez-Diaz, E., Rouillard, A. P., Davies, J. A. et al. 2017b, ApJ, 835, L7

Sanchez-Diaz, E., Rouillard, A. P., Lavraud, B. et al. 2019, ApJ, 882, 51

Sheeley, N. R., Wang, Y.-M., Hawley, S. H., et al. 1997, ApJ, 484, 472

Suess, S., Ko, Y.-K., Von Steiger, R., \& Moore, R. 2009, JGR, 114

Szabo, A., Larson, D. E., \& Lepping, R. P. 1999a, in AIP Conf. Proc. 471, Solar Wind Nine, ed. S. R. Habbal et al. (Melville, NY: AIP), 589 
Szabo, A., Larson, D., Whittlesey, P., et al. 2020, ApJS, 246, 47

Table 1. List of the eight true sector boundary intervals during orbit 1, with associated properties as observed from particle and magnetic field data. The intervals given merely correspond to those studied and that encompass the relevant density enhancements nearby the TSB.

\begin{tabular}{|c|c|c|c|c|c|c|}
\hline$\#$ & TSB interval studied & $\mathrm{N}$ increases & $\begin{array}{l}\text { Strahl } \\
\text { dropout }\end{array}$ & $\begin{array}{l}\text { Correlated } \\
\text { V-B changes* }\end{array}$ & Flux ropes & $\begin{array}{l}\mathrm{N} \text { increase and } \\
\text { dropout consistent }\end{array}$ \\
\hline 1 & $\begin{array}{l}2018-10-0912: 00 \\
2018-10-1100: 00\end{array}$ & Several & Yes & Yes & Maybe & Yes \\
\hline 2 & $\begin{array}{l}2018-10-1800: 00 \\
2018-10-1809: 00\end{array}$ & \multicolumn{5}{|c|}{$\begin{array}{l}\text { Insufficient resolution, but magnetic bifurcation possibly indicative of } \\
\text { reconnection at main current sheet. }\end{array}$} \\
\hline 3 & $\begin{array}{l}2018-10-2000: 00 \\
2018-10-2016: 00\end{array}$ & \multicolumn{5}{|c|}{ Complex HCS with TSB. Weak density signature and possible strahl dropout. } \\
\hline 4 & $\begin{array}{l}2018-10-27 \text { 20:00 } \\
2018-10-2907: 00\end{array}$ & Several & Yes & Yes & Yes & Yes \\
\hline 5 & $\begin{array}{l}2018-10-2907: 00 \\
2018-10-3007: 00\end{array}$ & Several & Yes & Yes & Yes & Yes \\
\hline 6 & $\begin{array}{l}2018-11-13 \text { 03:00 } \\
2018-11-1418: 00\end{array}$ & Several & Yes & Yes & Yes & Yes \\
\hline 7 & $\begin{array}{l}2018-11-23 \text { 10:00 } \\
2018-11-2404: 00\end{array}$ & Several & Yes & Yes & Maybe & Yes \\
\hline 8 & $\begin{array}{l}2018-12-0506: 00 \\
2018-12-0612: 00\end{array}$ & \multicolumn{5}{|c|}{ Complex HCS with TSB and insufficient resolution. } \\
\hline
\end{tabular}

402

403 

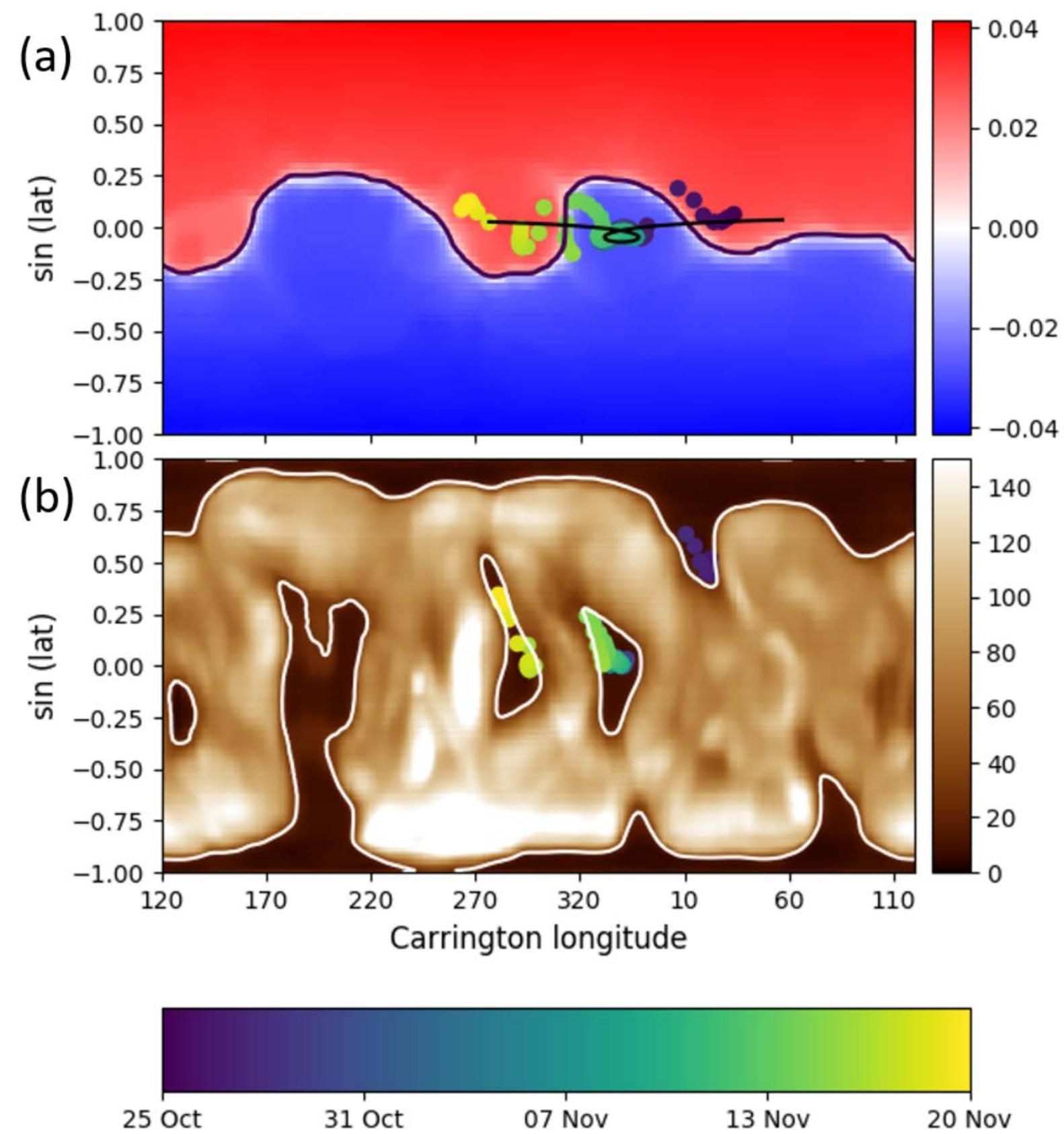

Figure 1. Carrington maps showing global modeling results of the solar corona and solar wind using the modeling performed by Réville et al. (2020). Panel (a) shows the radial magnetic field component at 5 Rs (in Gauss) together with the Heliospheric Current Sheet (HCS) as a thick black line. The PSP orbit magnetic mapping to $5 R_{s}$ is also shown as large colored points, on the basis of the global modelling results, as well as with a thin black line using a simple Parker spiral for comparison. Panel (b) displays the synthetic UV emission at $193 \AA$ from the corona (Digital Number units; cf. Boerner et al. (2012)), together with the magnetic mapping of PSP orbit to the photosphere within the global model. The orbit in both panels is colored according to the date during the orbit, as given in the color bar at the bottom. 


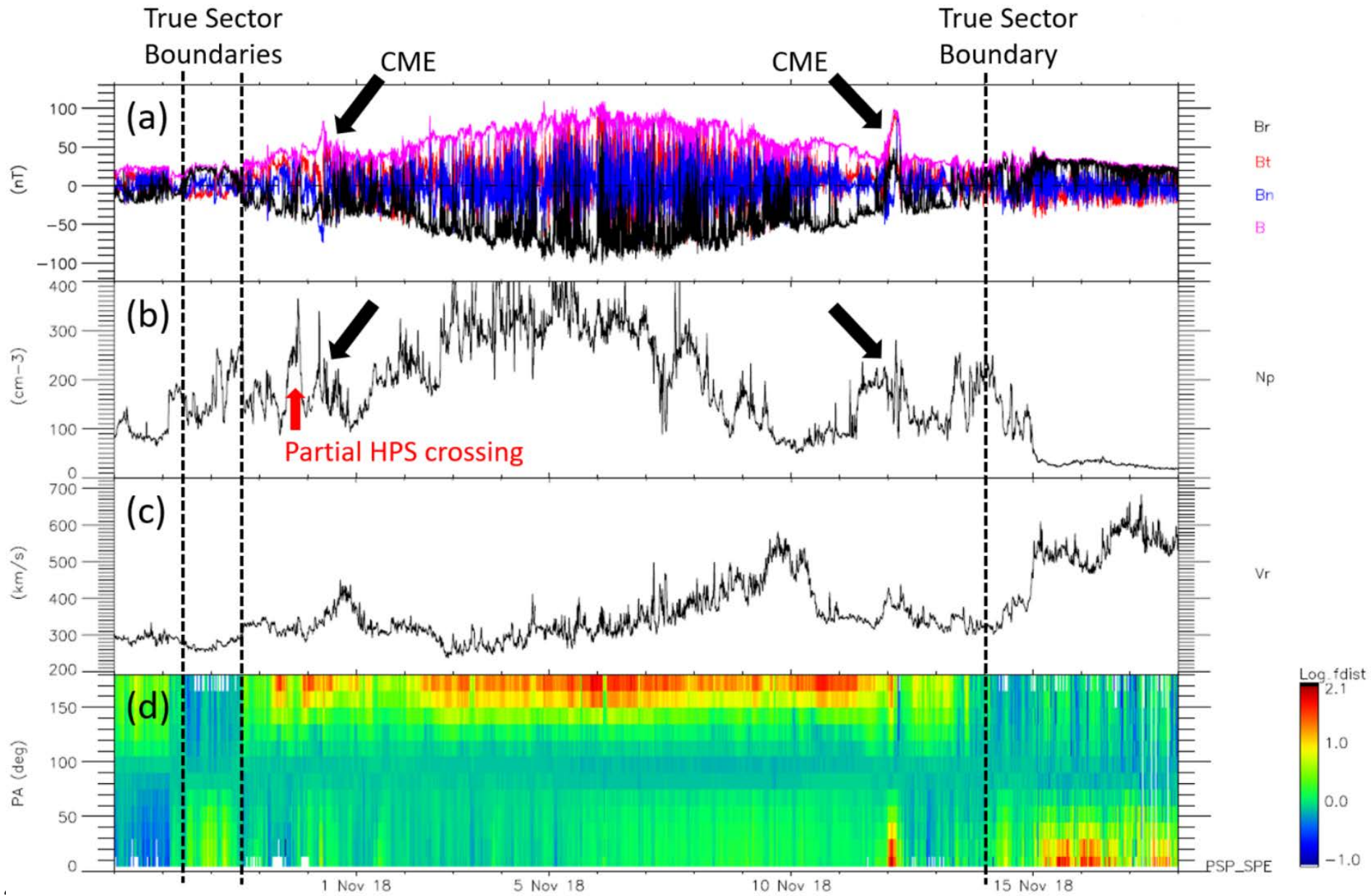

416 Figure 2. Parker Solar Probe observations from 27 October 2018 to 18 November 2018, 417 corresponding to the closest approach to the Sun during orbit 1. Panel (a) shows the magnetic 418 field vector and its magnitude. Panel (b) shows the ion density and panel (c) the ion radial 419 velocity component. Finally, panel (d) shows the $315 \mathrm{eV}$ suprathermal electron pitch angle 420 (PA) distributions in normalized unit; it shows the ratio of a given PA bin phase space density 421 (PSD) to the PSD at $90^{\circ} \mathrm{PA}$ for each sample (this unit is used given the very large dynamic range 422 of PSDs during the encounter, with color-coding according to the palette on the right-hand 423 side). The three vertical dashed lines mark the three sector boundaries observed during that 424 interval. There are density enhancements associated with them, as analyzed in the text. The 425 black arrows highlight the two main CMEs of orbit 1, with their associated density increase. A 426 small red arrow highlights another density increase, which is a partial crossing of the HCS and 427 is thus not listed in Table 1. 


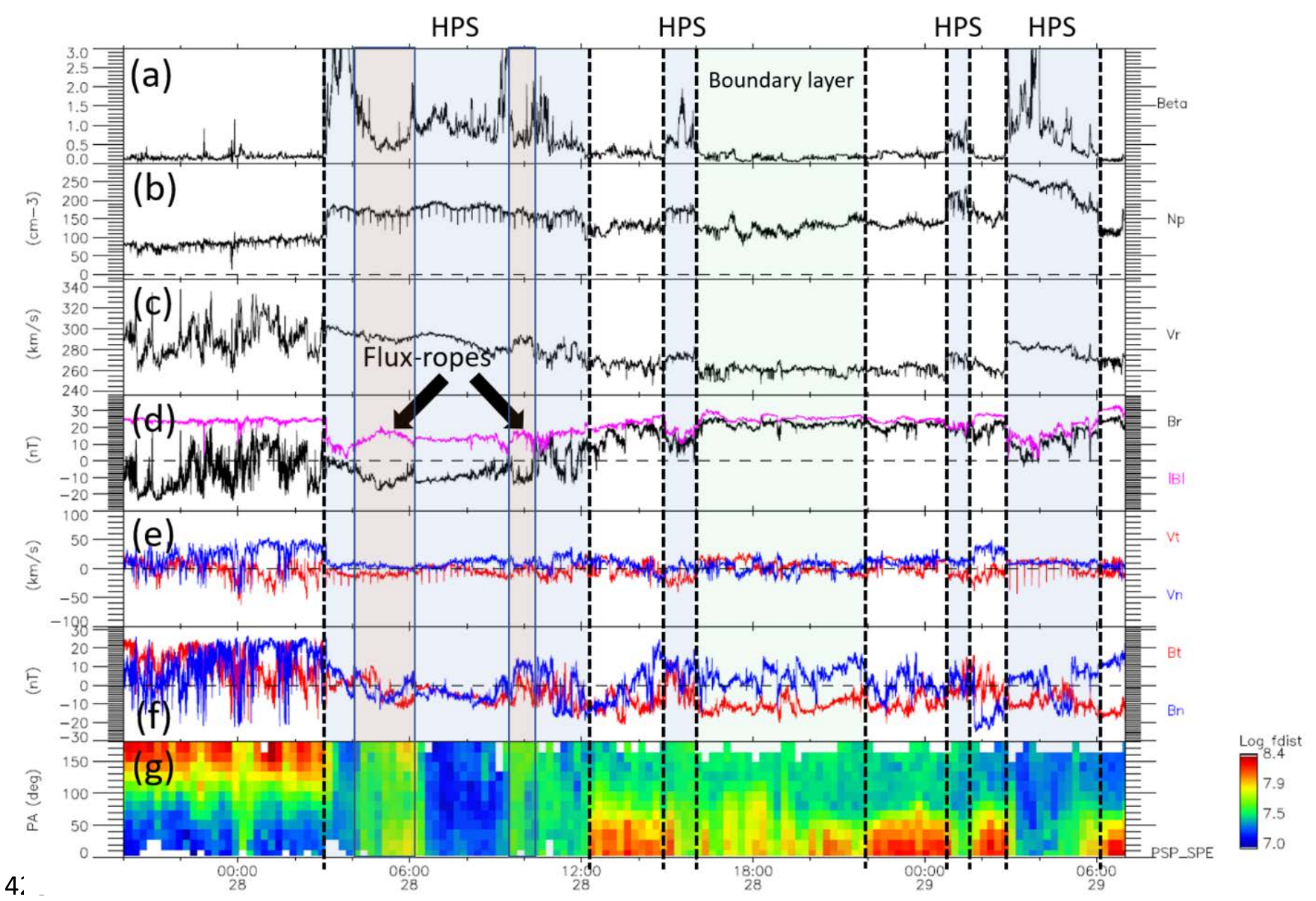

429 Figure 3. Parker Solar Probe observations from 22:00 UT on 27 October 2018 to 07:00 UT on 43029 October 2020. Panels (a) through ( $f$ ) show, respectively, the ion plasma $\beta$, density, $V_{R},|B|$ 431 and $B_{R}, V_{T}$ and $V_{N}$, and then $B_{T}$ and $B_{N}$. In panel (d) we highlight two possible flux rope 432 structures using black arrows and orange shading. Panel (g) shows $315 \mathrm{eV}$ suprathermal 433 electron pitch angle distributions, this time in actual PSD to best highlight strahl dropouts 434 (color-coding is according to the palette on the right-hand side). We highlight 4 HPS intervals 435 with blue shadowing, as well as what we call a boundary layer interval using green shadowing 436 (see section 4 for details). Note that a few spurious (wrong) data points appear mainly in the 437 density (panel (b)) and $\mathrm{V}_{\mathrm{T}}$ component (panel (e)), in particular during the first and last HPS 438 intervals highlighted. 


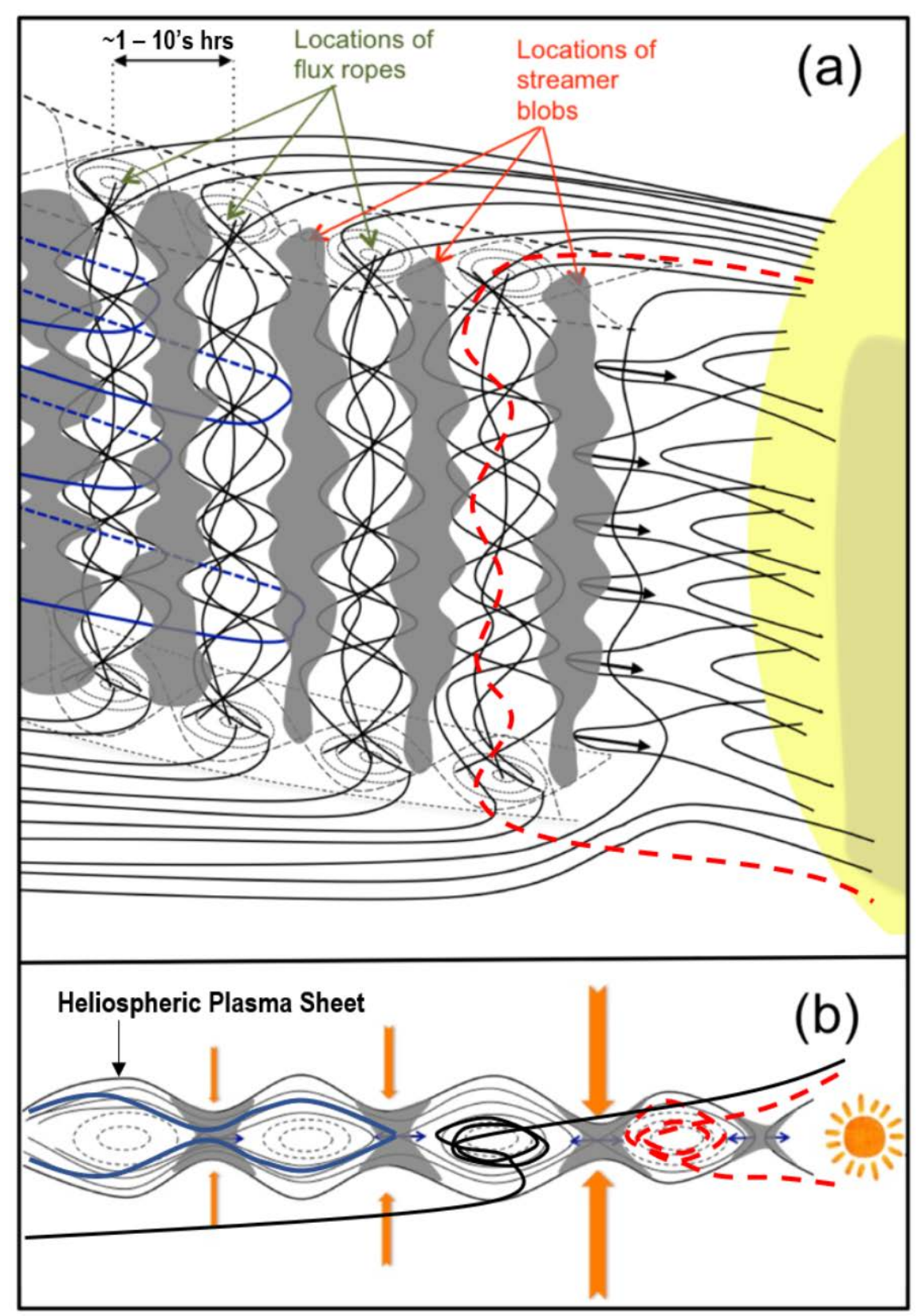

440 Figure 4. Sketch of magnetic reconnection as the origin of blobs in (a) a plane containing the neutral line and (b) a plane perpendicular to the neutral line. The gray areas indicate the location of the highest $\beta$ regions (or blobs in Sanchez-Diaz et al. (2019)). The black lines represent the magnetic field lines around the HCS. The dashed black lines represent the magnetic field lines structure in the vicinity of the flux ropes. While the black magnetic field lines that thread through all the flux ropes are here constructed such that they have only one end attached to the Sun, the red dashed line is meant to highlight that there can exist other configurations such that both ends may be connected to the Sun. Finally, the blue lines in panels ( $a$ ) and (b) show the magnetic field lines from the high- $\beta$ blobs, which surround the flux ropes, and that are typically disconnected from the Sun. The orange arrows in panel (b) show the inflows of magnetic reconnection at the $X$-lines formed by sequential magnetic reconnection at the tip of the helmet streamer. The small blue arrows in panel (b) show the exhaust velocities away from each $\mathrm{X}$-line. The figure is adapted from Sanchez-Diaz et al. (2019). 